\title{
RIESZ BASIS PROPERTY OF TIMOSHENKO BEAMS WITH BOUNDARY FEEDBACK CONTROL
}

\author{
DE-XING FENG, GEN-QI XU, and SIU-PANG YUNG
}

Received 18 January 2001 and in revised form 28 June 2001

\begin{abstract}
A Timoshenko beam equation with boundary feedback control is considered. By an abstract result on the Riesz basis generation for the discrete operators in the Hilbert spaces, we show that the closed-loop system is a Riesz system, that is, the sequence of generalized eigenvectors of the closed-loop system forms a Riesz basis in the state Hilbert space.
\end{abstract}

2000 Mathematics Subject Classification: 35C10, 47A65, 93B52, 93C20.

1. Introduction. The boundary feedback stabilization problem of a hybrid system has been studied extensively in the last decade. Many important results have been obtained. Among them, most of studies in the literatures are concerned with Euler-Bernoulli and Rayleigh beams; there are a few results for Timoshenko beams (cf. [3, 5, 6, 7, 9]), which are mainly focused on the stability of the closed-loop system. Though it is important to obtain the exponential stability of the system, it is also very interesting to study the rate of the exponential decay of the system. It is well known that if the system satisfies the spectrum-determined growth assumption, then the rate of the exponential decay can be easily estimated via the spectra of the system operator, see [2]. Furthermore, if the system is a Riesz one, that is, the set of the generalized eigenvectors of the system operator forms a Riesz basis of the state Hilbert space, then the spectrum-determined growth assumption is satisfied. In [1], the Riesz basis property was used to give some quantitative information of the rate of the exponential decay for a simpler Euler-Bernoulli beam system with no tip mass. For Euler-Bernoulli and Rayleigh beam systems, some further results concerning the Riesz basis property of the systems can be found in $[4,8]$.

In the present note, we consider the following Timoshenko beam equation with a tip mass (see $[7,9])$ :

$$
\begin{gathered}
\rho \ddot{w}(x, t)-K\left(w^{\prime \prime}(x, t)-\varphi^{\prime}(x, t)\right)=0, \quad 0<x<\ell, \\
I_{\rho} \ddot{\varphi}(x, t)-E I \varphi^{\prime \prime}(x, t)-K\left(w^{\prime}(x, t)-\varphi(x, t)\right)=0, \quad 0<x<\ell, \\
w(0, t)=0, \quad \varphi(0, t)=0, \\
M \ddot{w}(\ell, t)=-K\left(w^{\prime}(\ell, t)-\varphi(\ell, t)\right)+u_{1}(t), \\
J \ddot{\varphi}(\ell, t)=-E I \varphi^{\prime}(\ell, t)+u_{2}(t),
\end{gathered}
$$


with boundary feedback control

$$
\begin{gathered}
u_{1}(t)=-\alpha \dot{w}(\ell, t)-\gamma\left[\dot{w}^{\prime}(\ell, t)-\dot{\varphi}(\ell, t)\right], \\
u_{2}(t)=\beta \dot{\varphi}(\ell, t)-v \dot{\varphi}^{\prime}(\ell, t) .
\end{gathered}
$$

Here, $I_{\rho}, \rho, E I, K$, and $\ell$ are mass moment of inertia, mass density, rigidity coefficient, shear modulus of elasticity, and length of the beam, respectively, and $\alpha, \beta, \gamma$, and $\nu$ are positive feedback constants. Here, and henceforth, the dot and the prime denote derivatives with respect to time and space variables, respectively. In [9], the energy multiplier method is used to show the stability of the closed-loop system (1.1) with (1.2). In this note, the Riesz basis property of this system is proven, and hence the spectrum-determined growth assumption of the system is satisfied. Finally, based on this consideration, we show the exponential stability of the closed-loop system (1.1) with (1.2) via estimating the eigenvalues of $\mathcal{A}$.

2. The state space and eigenvalue problem. First, we recall the state space and the operator defined in [9]. Let

$$
\mathscr{H}=V_{0}^{1} \times L_{\rho}^{2}(0, \ell) \times V_{0}^{1} \times L_{I_{\rho}}^{2}(0, \ell) \times \mathbb{R} \times \mathbb{R},
$$

where $V_{0}^{k}=\left\{\varphi \in H^{k}(0, \ell) \mid \varphi(0)=0\right\}, k=1$, 2, with $H^{k}(0, \ell)$ the usual Sobolev space of order $k$. For $Y_{1}=\left[w_{1}, z_{1}, \varphi_{1}, \psi_{1}, \xi_{1}, \eta_{1}\right]^{T}, Y_{2}=\left[w_{2}, z_{2}, \varphi_{2}, \psi_{2}, \xi_{2}, \eta_{2}\right]^{T} \in$ $\mathscr{H}$, where the superscript $T$ denotes the transposition of a matrix, the inner product in $\mathscr{H}$ is defined by

$$
\begin{aligned}
\left\langle Y_{1}, Y_{2}\right\rangle= & \int_{0}^{\ell} K w_{1}^{\prime} \overline{w_{2}^{\prime}} d x+\int_{0}^{\ell} \rho z_{1} \overline{z_{2}} d x \\
& +\int_{0}^{\ell} E I \varphi_{1}^{\prime} \overline{\varphi_{2}^{\prime}} d x+\int_{0}^{\ell} I_{\rho} \psi_{1} \overline{\psi_{2}} d x+M^{-1} \xi_{1} \overline{\xi_{2}}+J^{-1} \eta_{1} \overline{\eta_{2}} .
\end{aligned}
$$

Define the linear operator $\mathscr{A}$ in $\mathscr{H}$ by

$$
\mathscr{A}\left(\begin{array}{c}
w \\
z \\
\varphi \\
\psi \\
\xi \\
\eta
\end{array}\right)=\left(\begin{array}{c}
z \\
\frac{K}{\rho}\left(w^{\prime \prime}-\varphi^{\prime}\right) \\
\psi \\
\frac{E I}{I_{\rho}} \varphi^{\prime \prime}+\frac{K}{I_{\rho}}\left(w^{\prime}-\varphi\right) \\
-\alpha z(\ell)-K\left(w^{\prime}(\ell)-\varphi(\ell)\right) \\
-\beta \psi(\ell)-E I \varphi^{\prime}(\ell)
\end{array}\right)
$$


with the domain

$$
\begin{aligned}
\mathscr{D}(\mathscr{A})=\left\{Y=[w, z, \varphi, \psi, \xi, \eta]^{T} \in \mathscr{H} \mid w, \varphi \in V_{0}^{2}, z, \psi \in V_{0}^{1},\right. \\
\left.\xi=M z(\ell)+\gamma\left(w^{\prime}(\ell)-\varphi(\ell)\right), \eta=J \psi(\ell)+v \varphi^{\prime}(\ell)\right\} .
\end{aligned}
$$

Then the closed-loop system (1.1) with (1.2) becomes the following evolution equation in $\mathscr{H}$ :

$$
\frac{d}{d t} Y(t)=\mathscr{A} Y(t), \quad \forall t>0
$$

where

$$
\begin{aligned}
Y(t)=[ & w(\cdot, t), \dot{w}(\cdot, t), \varphi(\cdot, t), \dot{\varphi}(\cdot, t), M \dot{w}(\ell, t) \\
& \left.+\gamma\left(w^{\prime}(\ell, t)-\varphi(\ell, t)\right), J \dot{\varphi}(\ell, t)+v \varphi^{\prime}(\ell, t)\right]^{T} .
\end{aligned}
$$

The following lemma can be found in [9].

LEMMA 2.1. The operator $A$ is dissipative and generates a $C_{0}$-semigroup with exponential decay.

In order to investigate the rate of the exponential decay of the closed-loop system (1.1) with (1.2), we study the Riesz basis property of the generalized eigenvector system of $\mathscr{A}$. For this purpose, we need the following lemma.

LEMMA 2.2. The operator $\mathscr{A}$ has compact resolvent on $\mathscr{H}$.

Proof. It follows from Lemma 2.1 that $0 \in \rho(\mathscr{A})$. Then for any $F=\left[f_{1}, f_{2}\right.$, $\left.g_{1}, g_{2}, \zeta_{1}, \zeta_{2}\right]^{T} \in \mathscr{H}$, there is a unique element $Y=[w, z, \varphi, \psi, \xi, \eta]^{T} \in \mathscr{H}$ such that $\mathscr{A} Y=F$, that is,

$$
\begin{gathered}
z=f_{1}, \quad \frac{K}{\rho}\left(w^{\prime \prime}-\varphi^{\prime}\right)=f_{2}, \quad \psi=g_{1}, \quad \frac{E I}{I_{\rho}} \varphi^{\prime \prime}+\frac{K}{I_{\rho}}\left(w^{\prime}-\varphi\right)=g_{2}, \\
-\alpha z(\ell)-K\left(w^{\prime}(\ell)-\varphi(\ell)\right)=\zeta_{1}, \quad-\beta \psi(\ell)-E I \varphi^{\prime}(\ell)=\zeta_{2},
\end{gathered}
$$

from which we obtain

$$
Y=\left(\begin{array}{c}
\int_{0}^{x} \varphi(s) d s-\frac{1}{K}\left(\alpha f_{1}(\ell)+\zeta_{1}\right) x+\frac{\rho}{K} \int_{0}^{\ell} k(x, s) f_{2}(s) d s \\
f_{1}(x) \\
\varphi(x) \\
g_{1}(x) \\
M f_{1}(\ell)-\frac{\gamma}{K}\left[\alpha f_{1}(\ell)+\zeta_{1}\right] \\
J \psi(\ell)+\frac{v}{E I}\left[\beta \psi(\ell)+\zeta_{2}\right]
\end{array}\right),
$$


where

$$
\begin{gathered}
\varphi(x)=-\frac{1}{E I}\left[\left(\beta g_{1}(\ell)+\zeta_{2}\right) x+I_{\rho} \int_{0}^{\ell} k(x, s) g_{2}(s) d s\right. \\
\left.+\left(\alpha f_{1}(\ell)+\zeta_{1}\right)\left(\ell x+\frac{x^{2}}{2}\right)+\rho \int_{0}^{\ell} k(x, s) d s \int_{s}^{\ell} f_{2}(r) d r\right], \\
k(x, s)= \begin{cases}s, & 0 \leq s \leq x, \\
x, & x \leq s \leq \ell .\end{cases}
\end{gathered}
$$

Then it is easy to see the compactness of $\mathscr{A}^{-1}$.

Now, we consider the eigenvalue problem of $\mathscr{A}$. Let $\lambda \in \mathbb{C}$ be an eigenvalue of $\mathscr{A}$ and $Y=[w, z, \varphi, \psi, \xi, \eta]^{T} \in \mathscr{H}$ an eigenvector corresponding to $\lambda$, then the functions $w(x)$ and $\varphi(x)$ satisfy

$$
\begin{gathered}
\rho \lambda^{2} w(x)-K\left(w^{\prime \prime}(x)-\varphi^{\prime}(x)\right)=0, \quad 0<x<\ell, \\
I_{\rho} \lambda^{2} \varphi(x)-E I \varphi^{\prime \prime}(x)-K\left(w^{\prime}(x)-\varphi(x)\right)=0, \quad 0<x<\ell, \\
w(0)=\varphi(0)=0, \\
\lambda^{2} M w(\ell)+K\left(w^{\prime}(\ell)-\varphi(\ell)=-\alpha \lambda w(\ell)-\lambda \gamma\left(w^{\prime}(\ell)-\varphi(\ell)\right),\right. \\
\left.\lambda^{2} J \varphi(\ell)+E I \varphi(\ell)=\beta \lambda \varphi(\ell)-\lambda v \varphi^{\prime}(\ell)\right) .
\end{gathered}
$$

Set

$$
\rho_{1}^{2}=\frac{\rho}{K}, \quad \rho_{2}^{2}=\frac{I_{\rho}}{E I}, \quad a=\rho_{1}^{2} \lambda^{2}, \quad b=\rho_{2}^{2} \lambda^{2}+\frac{K}{E I}, \quad c=-\frac{K}{E I} .
$$

Denote by $\mu_{1}$ and $\mu_{2}$ the two roots of the quadratic equation

$$
\mu^{2}-(a+b+c) \mu+a b=0
$$

that is,

$$
\begin{aligned}
& \mu_{1}=\frac{(a+b+c)+\sqrt{(a+b+c)^{2}-4 a b}}{2}, \\
& \mu_{2}=\frac{(a+b+c)-\sqrt{(a+b+c)^{2}-4 a b}}{2} .
\end{aligned}
$$


In the case of $\mu_{1} \neq \mu_{2}$, we define functions $w_{j}(\lambda, x)$ and $\varphi_{j}(\lambda, x)$ for $j=3,4$ by

$$
\begin{aligned}
& w_{3}(\lambda, x)=\frac{1}{\mu_{1}-\mu_{2}}\left(\left(\mu_{1}-b\right) \mu_{1}^{-1 / 2} \sinh \sqrt{\mu_{1}} x-\left(\mu_{2}-b\right) \mu_{2}^{-1 / 2} \sinh \sqrt{\mu_{2}} x\right), \\
& w_{4}(\lambda, x)=\frac{1}{\mu_{1}-\mu_{2}}\left(\cosh \sqrt{\mu_{1}} x-\cosh \sqrt{\mu_{2}} x\right), \\
& \varphi_{3}(\lambda, x)=\frac{1}{\mu_{1}-\mu_{2}} c\left(\cosh \sqrt{\mu_{1}} x-\cosh \sqrt{\mu_{2}} x\right), \\
& \varphi_{4}(\lambda, x)=\frac{1}{\mu_{1}-\mu_{2}}\left(\left(\mu_{1}-a\right) \mu_{1}^{-1 / 2} \sinh \sqrt{\mu_{1}} x-\left(\mu_{2}-a\right) \mu_{2}^{-1 / 2} \sinh \sqrt{\mu_{2}} x\right) .
\end{aligned}
$$

Set

$$
\begin{aligned}
& w(\lambda, x)=A w_{3}(\lambda, x)+B w_{4}(\lambda, x), \\
& \varphi(\lambda, x)=A \varphi_{3}(\lambda, x)+B \varphi_{4}(\lambda, x),
\end{aligned}
$$

where $A$ and $B$ are two constants to be determined. Then $w(\lambda, x), \varphi(\lambda, x)$ satisfy

$$
\begin{gathered}
\rho \lambda^{2} w(x)-K\left(w^{\prime \prime}(x)-\varphi^{\prime}(x)\right)=0, \quad 0<x<\ell, \\
I_{\rho} \lambda^{2} \varphi(x)-E I \varphi^{\prime \prime}(x)-K\left(w^{\prime \prime}(x)-\varphi^{\prime}(x)\right)=0, \quad 0<x<\ell, \\
w(0)=\varphi(0)=0, \quad w^{\prime}(0)=A, \quad \varphi^{\prime}(0)=B .
\end{gathered}
$$

From (2.10), we obtain

$$
\begin{aligned}
& A\left[\left(\lambda^{2} M+\alpha \lambda\right) w_{3}(\lambda, \ell)+(K+\gamma \lambda)\left(w_{3}^{\prime}(\lambda, \ell)-\varphi_{3}(\lambda, \ell)\right)\right] \\
& \quad+B\left[\left(\lambda^{2} M+\alpha \lambda\right) w_{4}(\lambda, \ell)+(K+\lambda \gamma)\left(w_{4}^{\prime}(\lambda, \ell)-\varphi_{4}(\lambda, \ell)\right)\right]=0, \\
& A\left[\left(\lambda^{2} J+\beta \lambda\right) \varphi_{3}(\lambda, \ell)+(E I+\lambda \nu) \varphi_{3}^{\prime}(\lambda, \ell)\right] \\
& \quad+B\left[\left(\lambda^{2} J+\beta \lambda\right) \varphi_{4}(\lambda, \ell)+(E I+\lambda \nu) \varphi_{4}^{\prime}(\lambda, \ell)\right]=0 .
\end{aligned}
$$

For an eigenpair $(\lambda, Y)$ of $\mathscr{A}$, the determinant $\Gamma(\lambda)$ of the coefficient matrix of the above linear equation system must be vanishing. Here

$$
\Gamma(\lambda)=\operatorname{det}\left[\begin{array}{ll}
\gamma_{11} & \gamma_{12} \\
\gamma_{21} & \gamma_{22}
\end{array}\right],
$$

where

$$
\begin{aligned}
& \gamma_{11}=\left(\lambda^{2} M+\alpha \lambda\right) w_{3}(\lambda, \ell)+(K+\lambda \gamma)\left(w_{3}^{\prime}(\lambda, \ell)-\varphi_{3}(\lambda, \ell)\right), \\
& \gamma_{12}=\left(\lambda^{2} M+\alpha \lambda\right) w_{4}(\lambda, \ell)+(K+\lambda \gamma)\left(w_{4}^{\prime}(\lambda, \ell)-\varphi_{4}(\lambda, \ell)\right), \\
& \gamma_{21}=\left(\lambda^{2} J+\beta \lambda\right) \varphi_{3}(\lambda, \ell)+(E I+\lambda \nu) \varphi_{3}^{\prime}(\lambda, \ell), \\
& \gamma_{22}=\left(\lambda^{2} J+\beta \lambda\right) \varphi_{4}(\lambda, \ell)+(E I+\lambda \nu) \varphi_{4}^{\prime}(\lambda, \ell) .
\end{aligned}
$$

Therefore, we have the following result. 
THEOREM 2.3. Assume that $w(\lambda, x), \varphi(\lambda, x)$, and $\Gamma(\lambda)$ are defined as before. Let $\lambda \in \mathbb{C}$ be such that $\mu_{1} \neq \mu_{2}$. Then $\lambda \in \sigma(\mathscr{A})$ if and only if $\Gamma(\lambda)=0$. In this case, an eigenvector of $A$ corresponding to $\lambda$ is

$$
Y=\left(\begin{array}{c}
w(\lambda, x) \\
\lambda w(\lambda, x) \\
\varphi(\lambda, x) \\
\lambda \varphi(\lambda, x) \\
M \lambda w(\lambda, \ell)+\gamma\left(w^{\prime}(\lambda, \ell)-\varphi(\lambda, \ell)\right) \\
J \lambda \varphi(\lambda, \ell)+v \varphi^{\prime}(\lambda, \ell)
\end{array}\right) .
$$

3. Riesz basis property of generalized eigenvector system of $\mathscr{A}$. In this section, we study the Riesz basis property of generalized eigenvector system of $\mathscr{A}$. We recall that the basis $\left\{\varphi_{n} \mid n \geq 1\right\}$ of a Hilbert space $\mathscr{H}$ is said to be a Riesz basis if it is equivalent to some orthonormal basis $\left\{e_{n} \mid n \geq 1\right\}$ of $\mathscr{H}$, that is, there is a bounded invertible linear operator $T$ on $\mathscr{H}$ such that $T \varphi_{n}=e_{n}$ for all $n \geq 1$. For the linear system (2.5), if the set of the generalized eigenvectors of the operator $\mathscr{A}$ forms a Riesz basis of the state Hilbert space $\mathscr{H}$, then the linear system (2.5) is called a Riesz system.

In the sequel, we prove that (2.5) is indeed a Riesz system. The following lemma can be found in [4].

LEMMA 3.1 [4]. Let $A$ be a densely defined discrete operator in a Hilbert space $\mathscr{H}$ and $\left\{\phi_{n} \mid n \geq 1\right\}$ a Riesz basis of $\mathcal{H}$. Assume that there are an integer $N_{1} \geq 0$ and a sequence of generalized eigenvectors $\left\{\psi_{n} \mid n>N_{1}\right\}$ of A such that

$$
\sum_{n=N_{1}+1}^{\infty}\left\|\phi_{n}-\psi_{n}\right\|^{2}<\infty .
$$

Then the following assertions hold.

(1) There are an integer $N_{2}>N_{1}$ and generalized eigenvectors $\left\{\tilde{\psi}_{n} \mid 1 \leq n \leq\right.$ $\left.N_{2}\right\}$ of A such that $\left\{\tilde{\psi}_{n} \mid 1 \leq n \leq N_{2}\right\} \cup\left\{\psi_{n} \mid n>N_{2}\right\}$ forms a Riesz basis of $\mathscr{H}$.

(2) Let $\left\{\tilde{\psi}_{n} \mid 1 \leq n \leq N_{2}\right\} \cup\left\{\psi_{n} \mid n>N_{2}\right\}$ correspond to eigenvalues $\left\{\sigma_{n} \mid\right.$ $n \geq 1\}$ of A. Then $\sigma(\mathscr{A})=\left\{\sigma_{n} \mid n \geq 1\right\}$, where each $\sigma_{n}$ is counted according to its algebraic multiplicity.

(3) If there is an integer $N_{3}>0$ such that $\sigma_{n} \neq \sigma_{m}$ for all $n, m>N_{3}$, then there is an integer $N_{4}>N_{3}$ such that any $\sigma_{n}$ for $n>N_{4}$ is algebraically simple.

From Lemma 3.1, it follows that in order to obtain the Riesz basis property of generalized eigenvector system of $\mathscr{A}$, we need to know some eigenvalues with corresponding eigenvectors of $\mathscr{A}$ and their asymptotic behavior.

Now, we discuss the asymptotic behavior of eigenvalues of $\mathscr{A}$. A lengthy computation shows that when $J-\rho_{2} v \neq 0$ and $M-\rho_{1} \gamma \neq 0$,

$$
\lim _{h \rightarrow-\infty} \inf _{\operatorname{Re} \lambda \leq h}|\Gamma(\lambda)|>0 .
$$


Thus, for $\lambda \in \sigma(\mathscr{A})$, it is sufficient to consider $\lambda$ lying in some vertical zone of complex plane, parallel to the imaginary axis. For $\lambda$ in this zone with $|\lambda|$ large enough, we have

$$
\begin{aligned}
& \frac{\Gamma(\lambda)}{\lambda^{2}} \\
& =\left|\begin{array}{cc}
\frac{M}{\rho_{1}} \sinh \ell \sqrt{\mu_{1}}+\gamma \cosh \ell \sqrt{\mu_{1}}+O\left(\lambda^{-1}\right) & O\left(\lambda^{-1}\right) \\
O\left(\lambda^{-1}\right) & \frac{J}{\rho_{2}} \sinh \ell \sqrt{\mu_{2}}+\nu \cosh \ell \sqrt{\mu_{2}}+O\left(\lambda^{-1}\right)
\end{array}\right| \\
& =0 .
\end{aligned}
$$

In the case of $J-\rho_{2} v \neq 0$ and $M-\rho_{1} \gamma \neq 0$, for $n \in \mathbb{Z}$, the set of all integers denote

$$
\begin{gathered}
\omega_{n}^{(1)}= \begin{cases}\frac{1}{2 \ell} \ln \left|\frac{M-\rho_{1} \gamma}{\rho_{1} \gamma+M}\right|+\frac{n \pi}{\ell} i, & \text { if } M>\rho_{1} \gamma, \\
\frac{1}{2 \ell} \ln \left|\frac{M-\rho_{1} \gamma}{\rho_{1} \gamma+M}\right|+\frac{(2 n+1) \pi}{2 \ell} i, & \text { if } M<\rho_{1} \gamma,\end{cases} \\
\omega_{n}^{(2)}= \begin{cases}\frac{1}{2 \ell} \ln \left|\frac{J-\rho_{2} v}{\rho_{2} v+J}\right|+\frac{n \pi}{\ell} i, & \text { if } J>\rho_{2} v, \\
\frac{1}{2 \ell} \ln \left|\frac{J-\rho_{2} v}{\rho_{2} v+J}\right|+\frac{(2 n+1) \pi}{2 \ell} i, & \text { if } J<\rho_{2} v .\end{cases}
\end{gathered}
$$

Then

$$
\begin{gathered}
M \sinh \ell \omega_{n}^{(1)}+\gamma \rho_{1} \cosh \ell \omega_{n}^{(1)}=0, \\
J \sinh \ell \omega_{n}^{(2)}+v \rho_{2} \cosh \ell \omega_{n}^{(2)}=0 .
\end{gathered}
$$

Set

$$
\lambda_{n}^{(1)}=\rho_{1}^{-1} \omega_{n}^{(1)}+\alpha_{n}^{(1)}, \quad \lambda_{n}^{(2)}=\rho_{2}^{-1} \omega_{n}^{(2)}+\alpha_{n}^{(2)},
$$

and let $\lambda_{n}^{(j)} \in \sigma(\mathscr{A})$ for $j=1,2$, then for $|n|$ large enough, we have $\alpha_{n}^{(j)}=$ $O\left(n^{-1}\right)$ for $j=1,2$.

Now, we consider the eigenvectors of $\mathscr{A}$. For $\lambda=\lambda_{n}^{(1)}$, take

$$
\begin{aligned}
& w^{\prime}(\lambda, 0)=\left(\lambda^{2} J+\lambda \beta\right) \varphi_{4}(\lambda, \ell)+(E I+\lambda v) \varphi_{4}^{\prime}(\lambda, \ell), \\
& \varphi^{\prime}(\lambda, 0)=-\left(\lambda^{2} J+\lambda \beta\right) \varphi_{3}(\lambda, \ell)-(E I+\lambda \nu) \varphi_{3}^{\prime}(\lambda, \ell),
\end{aligned}
$$

and for $\lambda=\lambda_{n}^{(2)}$, take

$$
\begin{aligned}
& w^{\prime}(\lambda, 0)=\left(\lambda^{2} M+\lambda \alpha\right) w_{4}(\lambda, \ell)+(K+\lambda \gamma) w_{4}^{\prime}(\lambda, \ell), \\
& \varphi^{\prime}(\lambda, 0)=-\left(\lambda^{2} M+\lambda \alpha\right) w_{3}(\lambda, \ell)-(K+\lambda \gamma) w_{3}^{\prime}(\lambda, \ell) .
\end{aligned}
$$


Set

$$
\begin{aligned}
& A_{n}=J \rho_{2}^{-1} \sinh \lambda_{n}^{(1)} \rho_{2} \ell+v \cosh \lambda_{n}^{(1)} \rho_{2} \ell \\
& B_{n}=M \rho_{1}^{-1} \sinh \lambda_{n}^{(2)} \rho_{1} \ell+v \cosh \lambda_{n}^{(2)} \rho_{1} \ell .
\end{aligned}
$$

Then with $|n|$ large enough, we have

$$
\begin{array}{ll}
w^{\prime}\left(\lambda_{n}^{(1)}, 0\right)=A_{n} \lambda_{n}^{(1)}+O(1), & \varphi^{\prime}\left(\lambda_{n}^{(1)}, 0\right)=O(1) \\
\varphi^{\prime}\left(\lambda_{n}^{(2)}, 0\right)=B_{n} \lambda_{n}^{(2)}+O(1), & w^{\prime}\left(\lambda_{n}^{(2)}, 0\right)=O(1) .
\end{array}
$$

For $\lambda \in \sigma(\mathscr{A})$ and $j=3,4$, denote

$$
Y_{j}(\lambda)=\left(\begin{array}{c}
w_{j}(\lambda, x) \\
\lambda w_{j}(\lambda, x) \\
\varphi_{j}(\lambda, x) \\
\lambda \varphi_{j}(\lambda, x) \\
M \lambda w_{j}(\lambda, \ell)+\gamma\left(w_{j}^{\prime}(\lambda, \ell)-\varphi_{j}(\lambda, \ell)\right) \\
J \lambda \varphi_{j}(\lambda, \ell)+v \varphi_{j}^{\prime}(\lambda, \ell)
\end{array}\right)
$$

then according to Theorem 2.3 , as an eigenvector of $\mathscr{A}$, we can take

$$
Y=\lambda^{-1} w^{\prime}(\lambda, 0) Y_{3}(\lambda)+\lambda^{-1} \varphi^{\prime}(\lambda, 0) Y_{4}(\lambda)
$$

Based on the above discussion, now we are able to prove the main result of the present note.

THEOREM 3.2. Let $\mathcal{H}$ and $\mathscr{A}$ be defined as before. If $J-\rho_{2} v \neq 0$ and $M-$ $\rho_{1} \gamma \neq 0$, then the generalized eigenvector system of A forms a Riesz basis of $\mathcal{H}$. Moreover, the eigenvalues of $A$ with large module are algebraically simple.

Proof. Assume, without loss of the generality, that $\rho_{1} \neq \rho_{2}$, then for $\lambda \in \mathbb{C}$ with $|\lambda|$ large enough, it follows that $\sqrt{\mu_{1}}=\rho_{1} \lambda+O\left(\lambda^{-1}\right)$ and $\sqrt{\mu_{2}}=\rho_{2} \lambda+$ $O\left(\lambda^{-1}\right)$. So, for $\lambda=\lambda_{n}^{(1)}$ with $|n| \geq N$, where $N$ is a sufficiently large positive integer, we have

$$
\begin{aligned}
& \sqrt{\mu_{1}}=\rho_{1} \lambda_{n}^{(1)}+O\left(n^{-1}\right)=\omega_{n}^{(1)}+O\left(n^{-1}\right), \\
& \sqrt{\mu_{2}}=\rho_{2} \lambda_{n}^{(1)}+O\left(n^{-1}\right)=\rho_{2} \rho_{1}^{-1} \omega_{n}^{(1)}+O\left(n^{-1}\right),
\end{aligned}
$$

and for $\lambda=\lambda_{n}^{(2)}$ with $|n| \geq N$, we have

$$
\begin{aligned}
& \sqrt{\mu_{1}}=\rho_{1} \lambda_{n}^{(2)}+O\left(n^{-1}\right)=\rho_{1} \rho_{2}^{-1} \omega_{n}^{(2)}+O\left(n^{-1}\right), \\
& \sqrt{\mu_{2}}=\rho_{2} \lambda_{n}^{(2)}+O\left(n^{-1}\right)=\omega_{n}^{(2)}+O\left(n^{-1}\right) .
\end{aligned}
$$


Therefore, for $\lambda_{n}^{(1)} \in \sigma(\mathscr{A})$ with $|n|$ large enough, we have

$$
Y_{3}\left(\lambda_{n}^{(1)}\right)=\left[\omega_{n}^{(1)^{-1}} \sinh \omega_{n}^{(1)} x, \rho_{1}^{-1} \sinh \omega_{n}^{(1)} x, 0,0,0,0\right]^{T}+G_{1}\left(\lambda_{n}^{(1)}\right),
$$

where $\left\|G_{1}\left(\lambda_{n}^{(1)}\right)\right\|=O\left(n^{-1}\right)$ and $Y_{4}\left(\lambda_{n}^{(1)}\right)=O(1)$.

Similarly, for $\lambda_{n}^{(2)} \in \sigma(\mathscr{A})$ with $|n|$ large enough, we have

$$
Y_{4}\left(\lambda_{n}^{(2)}\right)=\left[0,0, \omega_{n}^{(2)^{-1}} \sinh \omega_{n}^{(2)} x, \rho_{2}^{-1} \sinh \omega_{n}^{(2)} x, 0,0\right]^{T}+G_{2}\left(\lambda_{n}^{(1)}\right),
$$

where $\left\|G_{2}\left(\lambda_{n}^{(2)}\right)\right\|=O\left(n^{-1}\right)$ and $Y_{3}\left(\lambda_{n}^{(2)}\right)=O(1)$. Thus, it follows that

$$
Y\left(\lambda_{n}^{(1)}\right)=A_{n}\left[\omega_{n}^{(1)^{-1}} \sinh \omega_{n}^{(1)} x, \rho_{1}^{-1} \sinh \omega_{n}^{(1)} x, 0,0,0,0\right]^{T}+F_{1}\left(\lambda_{n}^{(1)}\right)
$$

with $\left\|F_{1}\left(\lambda_{n}^{(1)}\right)\right\|=O\left(n^{-1}\right)$, and that

$$
Y\left(\lambda_{n}^{(2)}\right)=B_{n}\left[0,0, \omega_{n}^{(2)}{ }^{-1} \sinh \omega_{n}^{(2)} x, \rho_{2}{ }^{-1} \sinh \omega_{n}^{(2)} x, 0,0\right]^{T}+F_{2}\left(\lambda_{n}^{(2)}\right)
$$

with $\left\|F_{2}\left(\lambda_{n}^{(2)}\right)\right\|=O\left(n^{-1}\right)$.

Noticing that

$$
0<\inf _{n \in \mathbb{Z}}\left|A_{n}\right|<\sup _{n \in \mathbb{Z}}\left|A_{n}\right|<\infty, \quad 0<\inf _{n \in \mathbb{Z}}\left|B_{n}\right|<\sup _{n \in \mathbb{Z}}\left|B_{n}\right|<\infty,
$$

it remains to prove that the sequences $\left\{\Phi_{n} \mid n \in \mathbb{Z}\right\} \cup\left\{\Psi_{n} \mid n \in \mathbb{Z}\right\}$, defined by

$$
\begin{aligned}
& \Phi_{n}=\left[\omega_{n}^{(1)^{-1}} \sinh \omega_{n}^{(1)} x, \rho_{1}^{-1} \sinh \omega_{n}^{(1)} x, 0,0,0,0\right]^{T}, \\
& \Psi_{n}=\left[0,0, \omega_{n}^{(2)^{-1}} \sinh \omega_{n}^{(2)} x, \rho_{2}{ }^{-1} \sinh \omega_{n}^{(2)} x, 0,0\right]^{T},
\end{aligned}
$$

form a Riesz basis of the subspace $\mathscr{H}_{1}$ of $\mathscr{H}$, where

$$
\mathscr{H}_{1}=\left\{Y \mid Y=[w, z, \varphi, \psi, 0,0]^{T} \in \mathscr{H}\right\} .
$$

Obviously, it is equivalent to prove that the sequence

$$
\left\{\left[\cosh \omega_{n}^{(1)} x, \rho_{1}^{-1} \sinh \omega_{n}^{(1)} x\right]^{T} \mid n \in \mathbb{Z}\right\}
$$


forms a Riesz basis of $L_{K}^{2}(0, \ell) \times L_{\rho}^{2}(0, \ell)$ and that the sequence

$$
\left\{\left[\cosh \omega_{n}^{(2)} x, \rho_{2}^{-1} \sinh \omega_{n}^{(2)} x\right]^{T} \mid n \in \mathbb{Z}\right\}
$$

forms a Riesz basis of $L_{E I}^{2}(0, \ell) \times L_{I_{\rho}}^{2}(0, \ell)$.

In the case of $M>\rho_{1} \gamma$, we define operator $\mathcal{T}$ by

$$
\mathscr{T}=\left(\begin{array}{cc}
\cosh \left(\frac{x}{2 \ell} \ln \left|\frac{M-\rho_{1} \gamma}{M+\rho_{1} \gamma}\right|\right) & \rho_{1} \sinh \left(\frac{x}{2 \ell} \ln \left|\frac{M-\rho_{1} \gamma}{M+\rho_{1} \gamma}\right|\right) \\
\rho_{1}^{-1} \sinh \left(\frac{x}{2 \ell} \ln \left|\frac{M-\rho_{1} \gamma}{M+\rho_{1} \gamma}\right|\right) & \cosh \left(\frac{x}{2 \ell} \ln \left|\frac{M-\rho_{1} \gamma}{M+\rho_{1} \gamma}\right|\right)
\end{array}\right),
$$

and in the case of $M<\rho_{1} \gamma$, we define

$$
\mathscr{T}=\left(\begin{array}{cc}
\cosh \left(\frac{x}{2 \ell}\left[\ln \left|\frac{M-\rho_{1} \gamma}{M+\rho_{1} \gamma}\right|+i \pi\right]\right) & \rho_{1} \sinh \left(\frac{x}{2 \ell}\left[\ln \left|\frac{M-\rho_{1} \gamma}{M+\rho_{1} \gamma}\right|+i \pi\right]\right) \\
\rho_{1}^{-1} \sinh \left(\frac{x}{2 \ell}\left[\ln \left|\frac{M-\rho_{1} \gamma}{M+\rho_{1} \gamma}\right|+i \pi\right]\right) & \cosh \left(\frac{x}{2 \ell}\left[\ln \left|\frac{M-\rho_{1} \gamma}{M+\rho_{1} \gamma}\right|+i \pi\right]\right)
\end{array}\right) .
$$

Obviously, $\mathcal{T}$ is a bounded invertible operator on $L_{K}^{2}(0, \ell) \times L_{\rho}^{2}(0, \ell)$ and satisfies

$$
\left(\begin{array}{c}
\cosh \omega_{n}^{(1)} x \\
\rho_{1}^{-1} \sinh \omega_{n}^{(1)} x
\end{array}\right)=\mathscr{T}\left(\begin{array}{c}
\cosh \frac{n \pi i x}{\ell} \\
\rho_{1}^{-1} \sinh \frac{n \pi i x}{\ell}
\end{array}\right) .
$$

Therefore, the sequence $\left\{\left[\cosh \omega_{n}^{(1)} x, \rho_{1}^{-1} \sinh \omega_{n}^{(1)} x\right]^{T} \mid n \in \mathbb{Z}\right\}$ forms a Riesz basis of $L_{K}^{2}(0, \ell) \times L_{\rho}^{2}(0, \ell)$ because the sequence

$$
\left\{\left[\cosh \frac{n \pi i x}{\ell}, \rho_{1}^{-1} \sinh \frac{n \pi i x}{\ell}\right]^{T} \mid n \in \mathbb{Z}\right\}
$$

is an orthonormal basis on $L_{K}^{2}(0, \ell) \times L_{\rho}^{2}(0, \ell)$.

The similar approach can be used to prove that the sequence

$$
\left\{\left[\cosh \omega_{n}^{(2)} x, \rho_{2}^{-1} \sinh \omega_{n}^{(2)} x\right]^{T} \mid n \in \mathbb{Z}\right\}
$$

forms a Riesz basis of $L_{E I}^{2}(0, \ell) \times L_{I_{\rho}}^{2}(0, \ell)$. Thus, the sequence $\left\{\Phi_{n} \mid n \in \mathbb{Z}\right\} \cup$ $\left\{\Psi_{n} \mid n \in \mathbb{Z}\right\}$ forms a Riesz basis of $\mathscr{H}_{1}$ and so does the sequence $\left\{A_{n} \Phi_{n} \mid n \in\right.$ $\mathbb{Z}\} \cup\left\{B_{n} \Psi_{n} \mid n \in \mathbb{Z}\right\}$. Therefore, there is a positive integer $N$ such that

$$
\sum_{|n| \geq N}^{\infty}\left\|Y\left(\lambda_{n}^{(1)}\right)-A_{n} \Phi_{n}\right\|^{2}+\sum_{|n| \geq N}^{\infty}\left\|Y\left(\lambda_{n}^{(2)}\right)-B_{n} \Psi_{n}\right\|^{2}<\infty .
$$

Using Lemma 3.1, the required result follows. 
According to the proof of Theorem 3.2, there exists an integer $N>0$ such that $\lambda_{n}^{(1)}, \lambda_{n}^{(2)}$ for $|n| \geq N$ are simple eigenvalues of $\mathscr{A}$, and $Y\left(\lambda_{n}^{(1)}\right)$ and $Y\left(\lambda_{n}^{(2)}\right)$ are two eigenvectors of $\mathscr{A}$ associated with $\lambda_{n}^{(1)}$ and $\lambda_{n}^{(2)}$, respectively. Denote

$$
\sigma_{0}=\sigma(\mathscr{A}) \backslash\left\{\lambda_{n}^{(1)}, \lambda_{n}^{(2)}|| n \mid \geq N\right\},
$$

then $\sigma_{0}$ is a finite set, that is,

$$
\sigma_{0}=\left\{\mu_{1}, \mu_{2}, \ldots, \mu_{k}\right\}
$$

with $k \leq 4 N-2$. For each eigenvalue $\mu_{j}$, let

$$
\left\{Y_{j, 1}, Y_{j, 2}, \ldots, Y_{j, s_{j}}\right\}
$$

be a basis of the corresponding root subspace. Therefore, by Theorem 3.2, the set

$$
\left\{Y_{j, i} \mid 1 \leq j \leq k, 1 \leq i \leq s_{j}\right\} \cup\left\{Y\left(\lambda_{n}^{(1)}\right)|| n \mid \geq N\right\} \cup\left\{Y\left(\lambda_{n}^{(2)}\right)|| n \mid \geq N\right\}
$$

forms a Riesz basis for $\mathscr{H}$. Let

$$
\left\{Y_{j, i}^{*} \mid 1 \leq j \leq k, 1 \leq i \leq s_{j}\right\} \cup\left\{Y^{*}\left(\lambda_{n}^{(1)}\right)|| n \mid \geq N\right\} \cup\left\{Y^{*}\left(\lambda_{n}^{(2)}\right)|| n \mid \geq N\right\}
$$

be the biorthogonal system associated with $\left\{Y_{j, i} \mid 1 \leq j \leq k, 1 \leq i \leq s_{j}\right\} \cup$ $\left\{Y\left(\lambda_{n}^{(1)}\right)|| n \mid \geq N\right\} \cup\left\{Y\left(\lambda_{n}^{(2)}\right)|| n \mid \geq N\right\}$. For each $F \in \mathscr{H}$, we have

$$
F=\sum_{j=1}^{k} \sum_{i=1}^{s_{j}}\left\langle F, Y_{j, i}^{*}\right\rangle Y_{j, i}+\sum_{|n| \geq N}\left\langle F, Y^{*}\left(\lambda_{n}^{(1)}\right)\right\rangle Y\left(\lambda_{n}^{(1)}\right)+\sum_{|n| \geq N}\left\langle F, Y^{*}\left(\lambda_{n}^{(2)}\right)\right\rangle Y\left(\lambda_{n}^{(2)}\right) .
$$

Let $T(t)$ be the $C_{0}$-semigroup generated by $\mathscr{A}$. Then, for each $Y \in \operatorname{span}\left\{Y_{j, i} \mid\right.$ $\left.i=1,2, \ldots, s_{j}\right\}$, we have

$$
T(t) Y=e^{\mu_{j} t} \sum_{i=1}^{s_{j}} P_{s_{j}, i}(Y, t) Y_{j, i},
$$

where $P_{s_{j}, i}(Y, t)$ is a polynomial of order less than $s_{j}$. Hence, we have the following corollary.

COROLlary 3.3. Let $\mathscr{H}$ and $A$ be as before. Let $T(t)$ be the $C_{0}$-semigroup generated by A. If $J-\rho_{2} v \neq 0$ and $M-\rho_{1} \gamma \neq 0$, then the solution of system (2.5) with initial data $F \in \mathscr{H}$ can be expressed as

$$
\begin{aligned}
T(t) F= & \sum_{j=1}^{k} \sum_{i=1}^{s_{j}} e^{\mu_{j} t} P_{s_{j}, i}(F, t) Y_{j, i}+\sum_{|n| \geq N} e^{\lambda_{n}^{(1)} t}\left\langle F, Y^{*}\left(\lambda_{n}^{(1)}\right)\right\rangle Y\left(\lambda_{n}^{(1)}\right) \\
& +\sum_{|n| \geq N} e^{\lambda_{n}^{(2)} t}\left\langle F, Y^{*}\left(\lambda_{n}^{(2)}\right)\right\rangle Y\left(\lambda_{n}^{(2)}\right) .
\end{aligned}
$$


4. Exponential decay of the closed-loop system. In Section 3, we discussed the Riesz basis property of system (2.5) in $\mathscr{H}$ and gave the solution expression of the system. In this section, we discuss the exponential decay of the closed-loop system. Since (2.5) is a Riesz system, according to [2], we have the following theorem.

THEOREM 4.1. Let $\mathcal{H}$ and $\mathscr{A}$ be as before. If $J-\rho_{2} \nu \neq 0$ and $M-\rho_{1} \gamma \neq 0$, then system (2.5) satisfies the spectrum-determined growth assumption in the state space $\mathcal{H}$.

Let $T(t)$ be the $C_{0}$-semigroup generated by $A$. The rate of exponential decay of $T(t)$ is defined by

$$
\omega(T)=\lim _{t \rightarrow \infty} \frac{\ln \|T(t)\|}{t}
$$

and the bound $s(\mathscr{A})$ of the spectrum of $\mathscr{A}$ is given by

$$
s(\mathscr{A})=\sup \{\operatorname{Re} \lambda \mid \lambda \in \sigma(\mathscr{A})\} .
$$

Theorem 4.1 implies that $\omega(T)=s(\mathscr{A})$. In order to estimate $s(\mathscr{A})$, denote

$$
\begin{aligned}
& \omega_{1}=\max \left\{\frac{1}{2 \ell \rho_{1}} \ln \left|\frac{M-\rho_{1} \gamma}{M+\rho_{1} \gamma}\right|, \frac{1}{2 \ell \rho_{2}} \ln \left|\frac{J-\rho_{2} v}{J+\rho_{2} v}\right|\right\}, \\
& \omega_{2}=\min \left\{\frac{1}{2 \ell \rho_{1}} \ln \left|\frac{M-\rho_{1} \gamma}{M+\rho_{1} \gamma}\right|, \frac{1}{2 \ell \rho_{2}} \ln \left|\frac{J-\rho_{2} v}{J+\rho_{2} v}\right|\right\} .
\end{aligned}
$$

From the discussion in Section 3, we know that the lines $\operatorname{Re} \lambda=\omega_{1}$ and $\operatorname{Re} \lambda=$ $\omega_{2}$ are two asymptotic lines of the spectrum of $\mathscr{A}$. Noticing that $\omega_{2}<\omega_{1}<0$, obviously for any $\varepsilon>0$ with $\omega_{1}+\varepsilon<0$, there are only finitely many eigenvalues of $\mathscr{A}$ outside the zone

$$
\omega_{2}-\varepsilon \leq \operatorname{Re} \lambda \leq \omega_{1}+\varepsilon .
$$

If $\operatorname{Re} \lambda \leq \omega_{1}$ for any $\lambda \in \sigma(\mathscr{A})$, then $s(\mathscr{A})=\omega_{1}$. If there is an eigenvalue $\lambda$ of $\mathscr{A}$ such that $\operatorname{Re} \lambda>\omega_{1}$, then there exists at least one eigenvalue $\lambda_{0}$ of $\mathscr{A}$ such that $\operatorname{Re} \lambda_{0}=s(\mathscr{A})$. Since $\mathscr{A}$ is a dissipative operator and $\sigma(\mathscr{A}) \cap i \mathbb{R}=\varnothing$, we have $s(\mathscr{A})<0$.

Summarizing the above discussion, we have the following result.

THEOREM 4.2. Let $\mathscr{A}$ and $\mathscr{H}$ be as before and $T(t)$ the $C_{0}$-semigroup of contractions generated by $\mathscr{A}$. Then it holds that

$$
\omega(T)=s(\mathscr{A})=\max _{\lambda \in \sigma(\mathscr{A})}\left\{\omega_{1}, \operatorname{Re} \lambda\right\}<0,
$$

and hence the closed-loop system is exponentially stable. 
We have proven that system (2.5) is a Riesz system. However, from the previous discussion we also can see that the asymptotic behavior of the eigenvalues of the closed-loop system operator $\mathscr{A}$ is dependent only upon the feedback parameters $\gamma$ and $\nu$. So, if we take $\alpha=0$ and $\beta=0$ in the feedback controls, then it is not difficult to prove that the corresponding closed-loop system is also a Riesz system. In this case, it is natural to ask whether the corresponding closed-loop system decays still exponentially. The answer is positive. In fact, if we denote by $\mathscr{A}_{1}$ the operator $\mathscr{A}$ with $\alpha=0$ and $\beta=0$, then $\mathscr{A}_{1}$ is also dissipative. In order to prove the exponential stability of the $C_{0}$-semigroup generated by $\mathscr{A}_{1}$, similar to above, it is enough to show that there is no eigenvalue of $\mathscr{A}_{1}$ on imaginary axis. It is easy to see that $0 \in \rho\left(\mathscr{A}_{1}\right)$. If $\lambda \in i \mathbb{R}$ is an eigenvalue of $\mathscr{A}_{1}$ and $Y=[w, z, \varphi, \psi, \xi, \eta]^{T} \in \mathscr{D}\left(\mathscr{A}_{1}\right)$ is an eigenvector of $\mathscr{A}_{1}$ corresponding to $\lambda$, then

$$
\operatorname{Re}\left\langle\mathscr{A}_{1} Y, Y\right\rangle=-\frac{\gamma K}{M}\left|w^{\prime}(\ell)-\varphi(\ell)\right|^{2}-\frac{\nu E I}{J}\left|\varphi^{\prime}(\ell)\right|^{2}=0,
$$

from which it follows that $w^{\prime}(\ell)-\varphi(\ell)=0$ and $\varphi^{\prime}(\ell)=0$. Then $w(x)$ and $\varphi(x)$ satisfy

$$
\begin{gathered}
\rho \lambda^{2} w(x)-K\left(w^{\prime \prime}(x)-\varphi^{\prime}(x)\right)=0, \quad 0<x<\ell, \\
I_{\rho} \lambda^{2} \varphi(x)-E I \varphi^{\prime \prime}(x)-K\left(w^{\prime}(x)-\varphi(x)\right)=0, \quad 0<x<\ell, \\
w(0)=\varphi(0)=0, \quad w^{\prime}(\ell)-\varphi(\ell)=0, \quad \varphi^{\prime}(\ell)=0, \\
w(\ell)=0, \quad \varphi(\ell)=0 .
\end{gathered}
$$

Thus, $w(\ell)=w^{\prime}(\ell)=0$ and $\varphi(\ell)=\varphi^{\prime}(\ell)=0$, and hence according to the general theory of ordinary differential equations, it follows that $w(x)=\varphi(x)=0$ for all $x \in[0, \ell]$, that is, $Y=0$. This shows that there is no eigenvalue of $\mathscr{A}_{1}$ on the imaginary axis. Notice the fact that when $J-\rho_{2} \nu \neq 0$ and $M-\rho_{1} \gamma \neq 0$, the lines $\operatorname{Re} \lambda=\omega_{1}$ and $\operatorname{Re} \lambda=\omega_{2}$ are two asymptotic lines of $\sigma\left(\mathscr{A}_{1}\right)$ and the system associated with $\mathscr{A}_{1}$ is a dissipative Riesz system; we can assert that the closed-loop system decays still exponentially and the rate of the exponential decay of the system is just equal to the supremum of real parts of the spectra of $\mathscr{A}_{1}$. Let $T_{1}(t)$ be the contraction semigroup generated by $\mathscr{A}_{1}$. Similar to above, we have the following result.

THEOREM 4.3. Let $\mathscr{A}_{1}$, He and $\omega_{1}$ be defined as before. Let $T_{1}(t)$ be the contraction semigroup generated by $\mathscr{A}_{1}$. Then it holds that

$$
\omega\left(T_{1}\right)=s\left(\mathscr{A}_{1}\right)=\max _{\lambda \in \sigma\left(\mathscr{A}_{1}\right)}\left\{\omega_{1}, \operatorname{Re} \lambda\right\}<0 .
$$

Notice that the exponential decay rates of the systems associated with $\mathscr{A}$ and $\mathscr{A}_{1}$ are different. In fact, we can show that $\omega(T) \leq \omega\left(T_{1}\right)$. It is very interesting to clarify the dependence of the exponential decay rate on the parameters $\alpha$ and $\beta$. 
ACKNOWLEDGMENTS. The authors would like to thank the referees for useful and helpful comments and suggestions. This research was supported by the National Natural Science Foundation of China (NSFC)-60174008, the Natural Science Foundation of Shanxi Province, and the HKRGC grant of code HKU 7133/02P.

\section{REFERENCES}

[1] F. Conrad and Ö. Morgül, On the stabilization of a flexible beam with a tip mass, SIAM J. Control Optim. 36 (1998), no. 6, 1962-1986.

[2] R. F. Curtain and H. Zwart, An Introduction to Infinite-Dimensional Linear Systems Theory, Texts in Applied Mathematics, vol. 21, Springer-Verlag, New York, 1995.

[3] D.-X. Feng, D.-H. Shi, and W. Zhang, Boundary feedback stabilization of Timoshenko beam with boundary dissipation, Sci. China Ser. A 41 (1998), no. 5, 483-490.

[4] B.-Z. Guo and R. Yu, The Riesz basis property of discrete operators and application to a Euler-Bernoulli beam equation with boundary linear feedback control, IMA J. Math. Control Inform. 18 (2001), no. 2, 241-251.

[5] K. Ito and N. Kunimatsu, Semigroup model and stability of the structurally damped Timoshenko beam with boundary inputs, Internat. J. Control 54 (1991), no. 2, 367-391.

[6] J. U. Kim and Y. Renardy, Boundary control of the Timoshenko beam, SIAM J. Control Optim. 25 (1987), no. 6, 1417-1429.

[7] Ö. Morgül, Boundary control of a Timoshenko beam attached to a rigid body: planar motion, Internat. J. Control 54 (1991), no. 4, 763-791.

[8] B. Rao, Optimal energy decay rate in a damped Rayleigh beam, Optimization Methods in Partial Differential Equations (South Hadley, Mass, 1996) (S. Cox and I. Lasiecka, eds.), Contemp. Math., vol. 209, American Mathematical Society, Rhode Island, 1997, pp. 211-229.

[9] D.-H. Shi, S. H. Hou, and D.-X. Feng, Feedback stabilization of a Timoshenko beam with an end mass, Internat. J. Control 69 (1998), no. 2, 285-300.

De-Xing Feng: Institute of Systems Science, Academy of Mathematics and Systems Science, Chinese Academy of Sciences, Beijing 100080, China

E-mail address: dxfeng@iss03.iss.ac.cn

Gen-Qi Xu: Department of Mathematics, Shanxi University, Taiyuan 030006, China

Siu-Pang Yung: Department of Mathematics, Faculty of Science, University of Hong Kong (HKU), Hong Kong

E-mail address: spyung@hkucc.hku.hk 


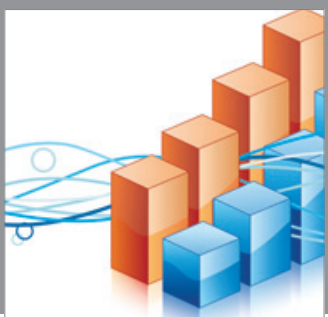

Advances in

Operations Research

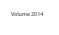

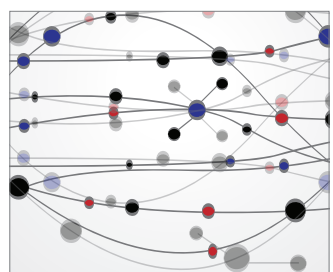

\section{The Scientific} World Journal
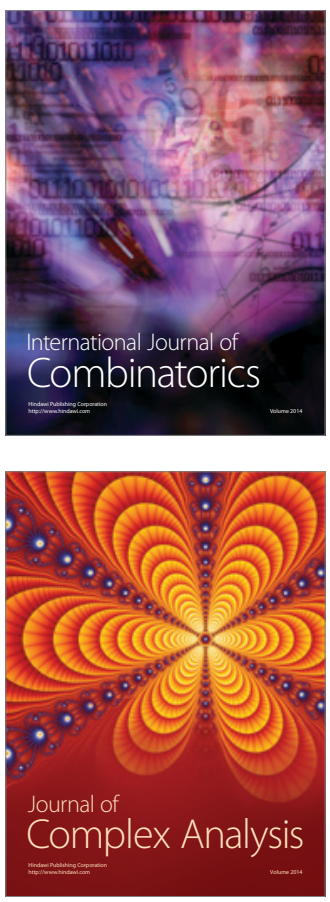

International Journal of

Mathematics and

Mathematical

Sciences
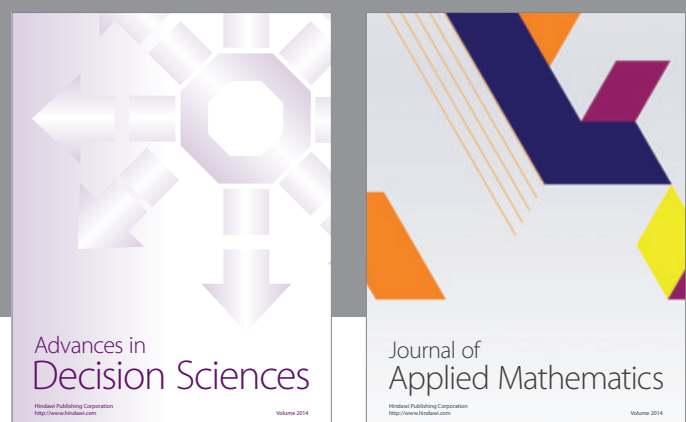

Journal of

Applied Mathematics
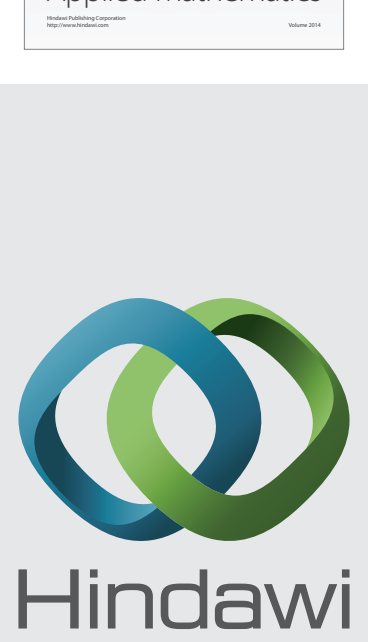

Submit your manuscripts at http://www.hindawi.com
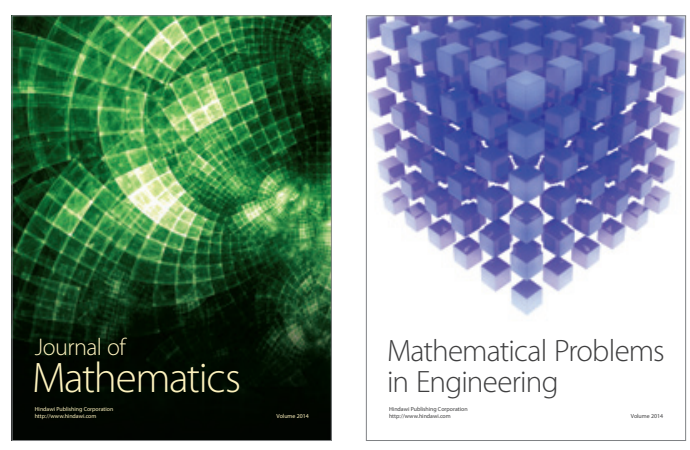

Mathematical Problems in Engineering
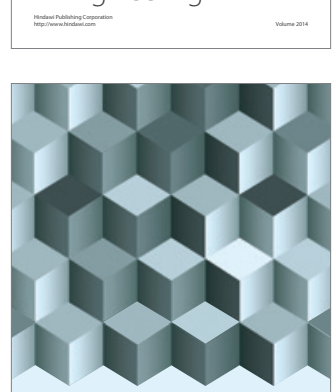

Journal of

Function Spaces
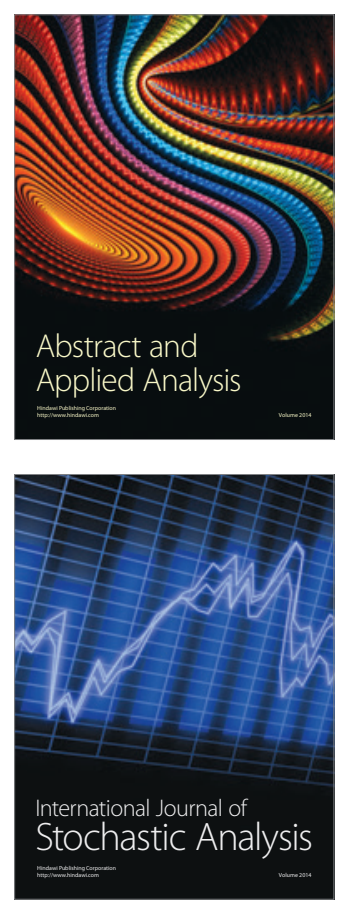

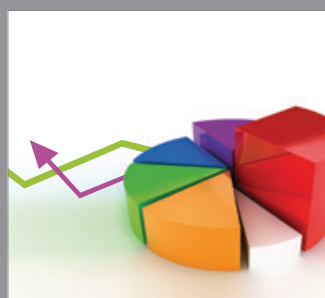

ournal of

Probability and Statistics

Promensencen
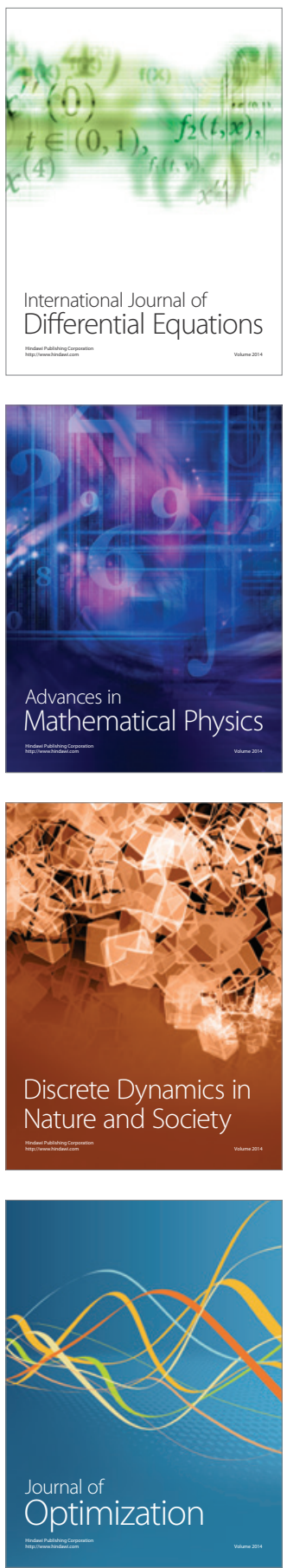